\title{
Experimental characterisation of the convective heat transfer in a vortex - wall interaction
}

\author{
by P. Reulet, M. Marchand and P. Millan
}

ONERAIDMAE unité ATAC 2, avenue Edouard Belin - BP 402531055 Toulouse Cedex 4 - France

\begin{abstract}
The development of turbulence models and wall laws for the numerical simulation of flows in complex geometries requires a detailed experimental analysis of turbulence and of the phenomena that appear in turbulent boundary layers. There is a strong need to develop new measurement systems allowing the determination of unsteady wall heat transfer coefficients.

In order to improve the knowledge of the unsteady phenomena occurring in perturbed boundary layers, a fundamental study is conducted on the interaction of a single vortex with a flat plate. An experimental methodology using a specific thermal sensor whose surface temperature is measured by an infrared thermography system is presented. It allows the characterisation of the unsteady convective heat transfer coefficient whose evolution is compared with the fluctuations of the wall friction coefficient, calculated from velocity profiles measured by laser Doppler velocimetry.
\end{abstract}

\section{Nomenclature}

$C_{c} \quad$ Specific heat of the ceramic

$C_{v} \quad$ Chord length of the wing

$C_{f} \quad$ Wall friction coefficient

$e_{c} \quad$ Ceramic layer thickness

$h_{C V} \quad$ Convective heat transfer coefficient

$k \quad$ Reduced frequency

$T_{P} \quad$ Wall temperature

$T_{a w} \quad$ Adiabatic wall temperature

$T_{C u} \quad$ Copper plate temperature

$T_{f} \quad$ Fluid temperature

$t \quad$ Time relative to the beginning of the wing motion

$U_{0} \quad$ Inlet velocity of the flow

$u \quad$ Horizontal velocity component

$v \quad$ Vertical velocity component

$x \quad$ Distance from the beginning of the thermal sensor (main flow direction)

$y \quad$ Lateral distance from the centreline of the thermal sensor

$z \quad$ Vertical distance from the wall $\alpha \quad$ Incidence angle of the airfoil

$\dot{\alpha} \quad$ Rotation velocity of the airfoil

$\varepsilon \quad$ Emissivity of the ceramic surface

$\gamma \quad$ Interaction parameter

$\varphi_{\text {total }}$ Total heat flux density (wall-fluid)

$\varphi_{\text {ray }}$ Radiative heat flux density

$\lambda_{c} \quad$ Thermal conductivity of the ceramic

$\mu \quad$ Dynamic viscosity of the fluid

$\rho \quad$ Density of the fluid

$\rho_{c}$

$\tau_{p}$

$\omega$

Re

Pr

Density of the ceramic

Wall shear stress

Pulsation frequency

Reynolds number

PrandtI number

\section{Introduction}

The study of perturbed boundary layers is fundamental for the understanding of the heat transfer phenomena between a fluid and a wall and their correlation with the dynamic characteristics of the flow near the wall. That is the reason why this study has been conducted: to characterise the unsteady convective heat transfer induced by the interaction between a vortex and a laminar boundary layer.

The dynamic analysis of the interaction is carried out in two phases: a global determination of the velocity field to analyse the evolution of the vortex followed by a more detailed analysis of the boundary layer. The latter allows the evaluation of the wall friction coefficient, the evolution of which will be compared to the unsteady convective heat transfer coefficient. 


\section{Experimental setup and procedure}

The coupled dynamic and thermal study of the vortex - wall interaction requires the generation of a single, reproducible eddy with known characteristics (circulation, viscous radius, convection velocity). A wall, on which a laminar boundary layer develops, is also defined. These two systems are integrated in a closed wind-tunnel. The test section is presented in figure 1.

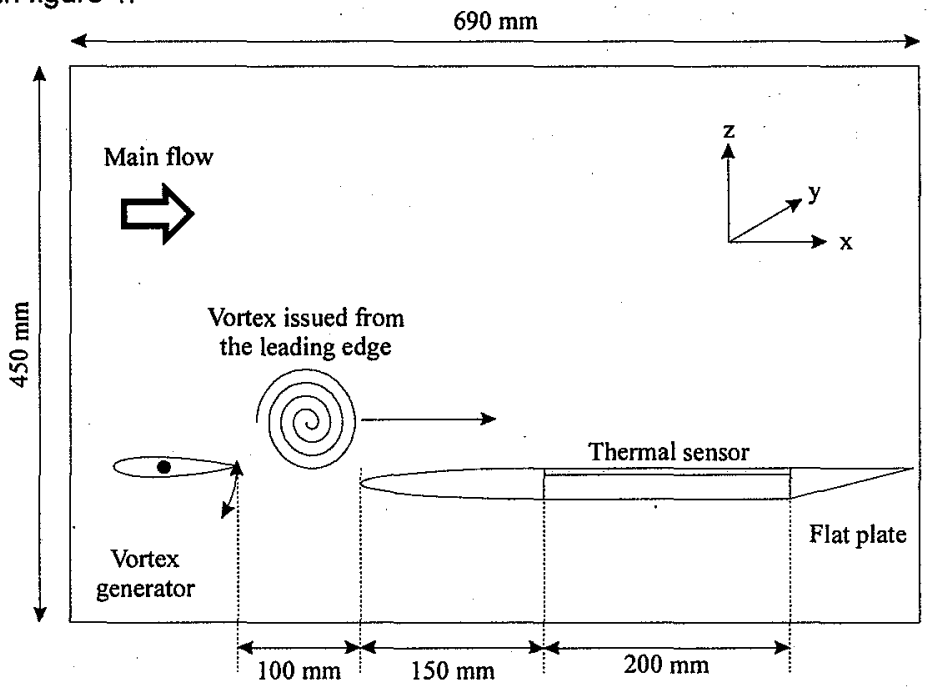

Fig. 1. Schematic of the test section

\subsection{Experimental apparatus}

\subsubsection{The vortex generator}

The objective is to generate a negative circulation vortex with its rotation axis perpendicular to the mainstream. As shown in the literature, the generation of an eddy is controlled by several parameters of the vartex generator such as $C_{v}$ (the wing chord), $\alpha$ (the rotation angle), $\dot{\alpha}$ (the rotation velocity for a non-sinusoidal motion), $\omega$ (the oscillation pulsation for a sinusoidal motion) and $k$ (the reduced frequency) defined both for sinusoidal and non-sinusoidal motions as :

$$
k=\frac{\omega C_{V}}{2 U_{0}} \text { for sinusoidal motion or } k=\frac{\dot{\alpha} C_{V}}{2 U_{0}} \text { for non-sinusoidal motion }
$$

Among the works on the subject is the experimental study of Booth and $Y u$ [1], concerning the generation of a von Karman street of vortices by a sinusoidal oscillation of a wing. This principle, using a non-sinusoidal motion, has also been used by Walker et al. [2]. It enables the generation of a single vortex. The main parameter governing the generation is the reduced frequency. A parametric study has then been made by Francis and Keesee [3] for profiles of different geometries and different chord-length-based Reynolds numbers $\left(\operatorname{Re}_{C_{r}}\right)$.

Then, Peube and Ferret [4] proposed to generate a single vortex by the rapid motion of a NACA wing in the main flow: the wing is first quickly brought to a maximum incidence angle, which creates an eddy on its upper surface. After a short time at the maximum 
incidence, the wing is brought back to zero incidence. Indeed, this permits the ejection of the vortex in the main flow.

So the system of vortex generation that has been presently chosen is based on the rapid rotation of a NACA 0015 airfoil, around its axis. The motion is controlled by a pneumatic actuator connected to an electronic relay, allowing to set the air inlet and outlet and the duration of a wing motion (about $0.2 \mathrm{~s}$ ). Furthermore, the "beating" of the NACA wing is governed by a frequency generator connected to the relay and set to a value of $2 \mathrm{~Hz}$ assuring the independence of two successive vortexes.

\subsubsection{The flat plate}

In order to generate a laminar boundary layer, an aluminium flat plate (figure 2) is placed downstream of the NACA wing and occupies the entire width of the wind tunnel test section so as to prevent boundary layer effects on its lateral sides.

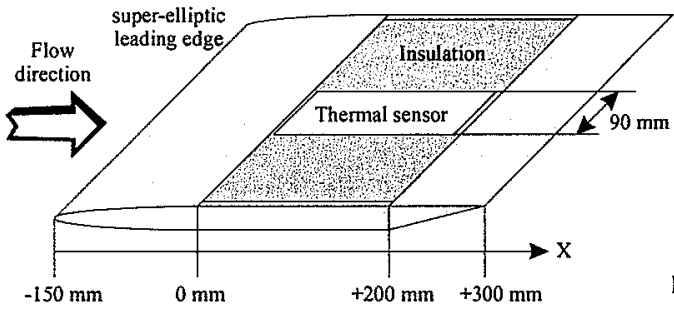

Fig. 2. Flat plate including the thermal sensor

The leading edge of the plate has a super-elliptic shape, allowing a laminar boundary layer to develop (if the main flow turbulence level is sufficiently low), and preventing the separation of the boundary layer by controlling the pressure gradient. The dimensions of the plate are designed so as to include the heated thermal sensor which is used to determine the convective unsteady heat transfer coefficient occurring when the vortex passes above the plate.

\subsection{Measurement techniques}

\subsubsection{Laser Doppler velocimetry}

A TSI 2-dimensional fringe laser anemometer coupled to a $4 \mathrm{~W}$ Argon laser beam is used to perform the measurements of the two components $u$ ( $x$ direction) and $v$ ( $z$ direction) of the instantaneous velocity. The air is seeded with incense particles of about $1 \mu \mathrm{m}$ size. The backscattering light is detected by the photomultipliers by means of a multimode optic fibre and then directed as an electric signal to the data acquisition system.

A specific treatment of these measurements is developed to reconstruct the instantaneous velocity fields over one period of the wing motion. It uses the simultaneous acquisition of the two components of the velocity and of the signal that controls the wing motion. The detection of the beginning of each motion permits the calculation of the mean velocity and its fluctuations (representing the turbulence intensity) for each measurement location at a given time.

\subsubsection{Measurement of the wall heat transfer coefficient}

The identification of the wall heat transfer is based on the use of a specific thermal sensor. A thin isolating ceramic layer is exposed to the flow on one face and is heated at the other one through a copper plate (figure 2). The ceramic is chosen for its low thermal inertia, its properties are: 


$$
\begin{aligned}
& \lambda_{c}=0.033 \mathrm{~W} / \mathrm{m} \cdot K \\
& \rho_{c}=190 \mathrm{~kg} / \mathrm{m}^{3} \\
& C_{c}=1045 \mathrm{~J} / \mathrm{kg} \cdot K
\end{aligned}
$$

Indeed if a high frequency thermal perturbation occurs on its surface, the thermal penetration thickness is very small and so is the thermal inertia of the involved layer. The ceramic is also chosen because of its high emissivity $(\varepsilon=0.89)$ in the spectral band 8-12 $\mu \mathrm{m}$ which is used for the infrared thermography measurement of its surface temperature.

\subsubsection{Mean heat transfer identification}

To measure the mean heat transfer coefficient, a sequence of temperature maps is acquired by the infrared thermography system in steady state conditions. The mean temperature is then calculated at each measurement point of the field. Due to the geometry of the sensor, some hypothesis can be made in order to develop a simple identification method. First, the lateral heat conduction in the insulating ceramic layer can be neglected. The copper plate is thick enough to assume that the temperature is constant all over the back surface of the ceramic layer. Therefore, the convective heat transfer coefficient can be calculated at each measurement point by a onedimensional heat balance :

$$
\frac{\lambda_{c}}{e_{c}}\left(T_{C u}-T_{p}(x, y)\right)=h_{C V}(x, y) \cdot\left(T_{p}(x, y)-T_{a w}(x, y)\right)+\varphi_{r a y}(x, y)
$$

For our range of velocity, the adiabatic wall temperature is equal to the temperature of the flow $T_{a w}=T_{f l}$. To evaluate the radiative heat flux density, the walls of the test section are assumed to behave like a black body at the temperature of the flow:

$$
\varphi_{\text {ray }}(x, y)=\varepsilon \sigma\left(T_{P}^{4}(x, y)-T_{n}^{4}\right)
$$

\subsubsection{Unsteady heat transfer identification}

The calculation method for the identification of the unsteady convective heat transfer coefficient is adapted to the acquisition modes of the infrared thermography system. Because of the high acquisition rate herein needed, only a temperature profile along the flow direction is measured. A simulation code resolving the heat equation for a twodimensional multilayer medium has been developed. The heat equation is discretised with a CrankNicholson scheme and the resulting system is solved using the ADI method. This scheme is unconditionally stable, second order accurate in space and time.

In the present study, only the ceramic layer is treated (figure 3), with a constant temperature on its lower face (temperature measured by thermocouples inside the copper plate), the lateral faces are supposed thermally isolated. The evolution of the temperature on the upper face is given by the infrared thermography measurements.

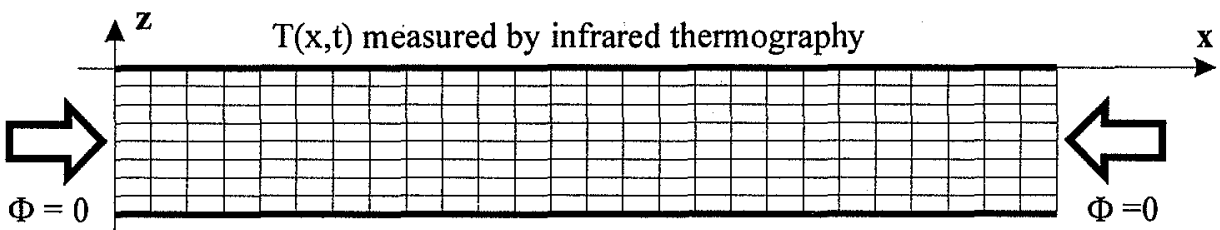

Temperature inside the copper plate

Fig. 3. Calculation domain and boundary conditions

This simulation code calculates the heat flux distribution at the fluid - wall interface, from which the convective heat transfer coefficient is determined by the equation: 


$$
\varphi_{\text {total }}(x, t)=h_{C V}(x, t) \cdot\left(T_{p}(x, t)-T_{n}\right)+\varphi_{\text {ray }}(x, t)
$$

The radiative heat flux density is calculated in the same manner as in the steady state case:

$$
\varphi_{\text {ray }}(x, t)=\varepsilon \sigma\left(T_{p}^{4}(x, t)-T_{j}^{4}\right)
$$

A second acquisition system records the signal that controls the wing motion so as to enable to calculate a synchronic average in order to reduce the noise present in the temperature measurements.

Further details can be found in Reulet et al. [5].

\section{Results}

Concerning the interaction of the vortex with a wall, the only studies that the present authors are aware of are numerical ones.

Doligalski and Walker [6] made a numerical study for a non heated plate. An important parameter was the interaction parameter $\gamma$, the ratio between the convection velocity of the vortex and the mainstream velocity, which ranged from 0 to 1 . Different phenomena have been observed depending on the value of $\gamma$ :

- $\gamma<0.75$ : the boundary layer is thickened downstream of the vortex and a secondary vortex is formed;

- $\gamma>0.75$ : the thickening of the boundary layer appears upstream the vortex.

Escriva [7] has studied numerically the influence of the vortex issued from a backward facing step with a heated flat plate. From a dynamic point of view, this author observes a recirculation zone downstream of the step and the presence of a reattachment point characterising the re-establishment of the boundary layer.

\subsection{Experimental conditions}

The first steps of this study, concerning the experimental conditions for the creation of a single vortex with suitable characteristics are presented in the article of Reulet et al. [8]. This paper describes the dynamic characterisation of each system: the vortex and the boundary layer. The large eddy issued from the leading edge of the wing has been investigated to determine its circulation, viscous radius and convection velocity. The boundary layer is laminar-like without any outer perturbation. But, for the study of the interaction, the plate must be placed in the wake of the wing where the velocity profiles of the boundary layer are turbulent.

The present paper deals with the characterisation of the interaction between these two systems.

The main experimental conditions are:

- Inlet velocity: $U_{0}=3.2 \mathrm{~m} / \mathrm{s}$

- Reynolds number based on the wing chord: $\operatorname{Re}_{C_{r}}=20400$

- Maximum angle of attack of the wing: $\alpha_{\max }=30^{\circ}$

- Reduced frequency: $0<k<0.9$

\subsection{Aerodynamic analysis}

\subsubsection{Global velocity and rotational fields}

A first measurement campaign of the complete test section above the flat plate is conducted by laser Doppler velocimetry. The processing of these measurements permits the reconstruction of the instantaneous velocity fields, regularly time-spaced over one period of 
the wing motion (cf. 2.2.1). From these velocity fields, the rotational fields are calculated to detect more precisely the vortex.

Figure 4 shows the organised structure of the vortex at a given time. The velocity field is plotted after the suppression of the mean velocity field calculated over the first $50 \mathrm{~ms}$ during which the flow is not perturbed. The vortex keeps a well-organised structure all along the flat plate even though its own induced velocities are opposed to those of the main flow near the wall.
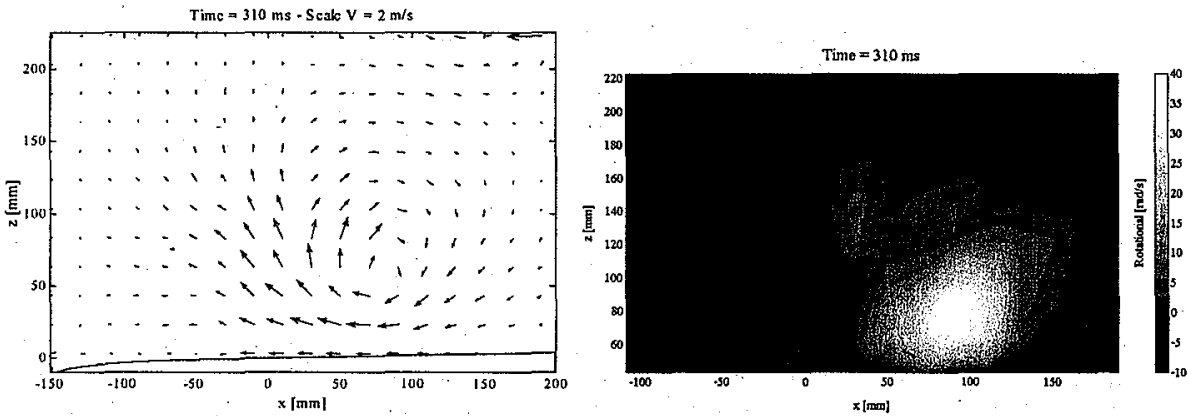

Fig. 4. Instantaneous velocity and rotational field

The rotational fields confirm these observations. The centre of the eddy follows a rising trajectory and its intensity decreases rapidly, because of the viscous dissipation at the wall. Another interesting phenomenon that is not detected on the velocity fields appears: a second contra-rotating structure forms just after the occurrence of the main vortex. However its intensity is rather small and its presence only seems due to the rapid rotation of the wing going back to zero incidence.

From the successive rotational fields, the approximate position of the vortex centre is determined. The following table gives the occurrence times of the vortex centre at each abscissa where the boundary layer will be investigated (next paragraph) :

\begin{tabular}{|c|c|c|c|c|c|}
\hline Position of the vortex centre: $\mathbf{x}[\mathrm{mm}]$ & 0 & 50 & 100 & 150 & 200 \\
\hline Time [s] & 0.272 & 0.292 & 0.311 & 0.331 & 0.350 \\
\hline
\end{tabular}

\subsubsection{Dynamic characterisation of the perturbed boundary layer}

A more detailed study of the interaction requires precise velocity measurements inside the: boundary layer developing on the flat plate. So, a second measurement campaign is conducted in a region $30 \mathrm{~mm}$ thick, above the thermal sensor. Only the first profile (at $x=0 \mathrm{~mm}$ ) is presented, at different times (figure 5).

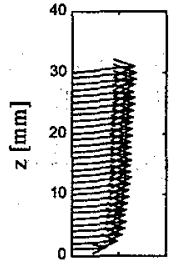

$0-50[\mathrm{~ms}]$

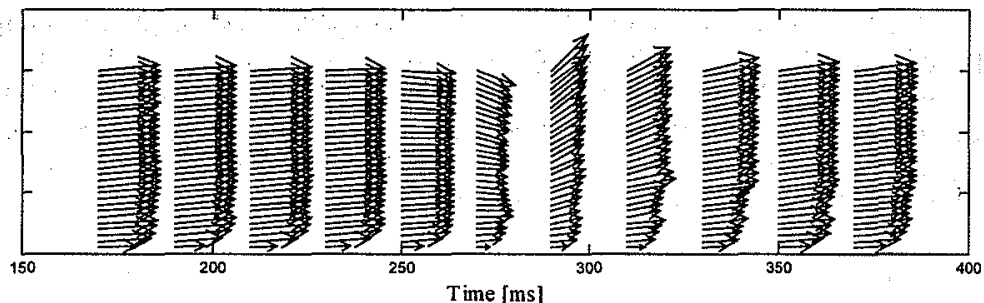

Time [ms]

Fig. 5. Instantaneous velocity profiles in the boundary layer $(x=0 \mathrm{~mm})$ 
The first profile represents the mean velocity from 0 to $50 \mathrm{~ms}$ : it shows the structure of the boundary layer in the wake of the wing. The other profiles are equally spaced over the passage of the vortex above the thermal sensor.

The phenomena that appear here can be decomposed:

- Until $t=100 \mathrm{~ms}$, the flow is not yet perturbed by the vortex.

- From $t=180 \mathrm{~ms}$, the flow is accelerated and the boundary layer profiles take a laminar-like shape.

- At $t=250 \mathrm{~ms}$, the influence of the vortex appears, the velocity decreases and the vectors are inclined towards the plate: the boundary layer is highly perturbed.

- At $t=270 \mathrm{~ms}$, the vortex is centred above the position $x=0 \mathrm{~mm}$, the velocities at this position are minimal and the boundary layer is compressed.

- At $t=290 \mathrm{~ms}$, just after the passage of the vortex, the boundary layer seems to be stretched up, but no separation occurs.

- Then, the flow slowly comes back to its initial situation.

As the vortex is convected downstream, the same observations are valid at each abscissa on the plate for later times. These effects are smaller as the vortex intensity decreases.

The relaminarisation of the boundary layer just before the passage of the vortex was verified by the visualisation of the flow using a fast video camera. Indeed, before the rotation of the wing, it is possible to distinguish the small eddies constituting its wake which roll over the flat plate and induce a turbulent-like boundary layer profile. When the wing is at maximum incidence, these small eddies issued from the trailing edge are convected under the flat plate, and so the boundary layer can assume to its laminar state just before the passage of the principal vortex. This result is also confirmed by the evolution of the instantaneous turbulence intensity on the first point of each profile: a clear diminution occurs that indicates a relaminarisation. The corresponding times when this phenomenon appears are given in the following table:

\begin{tabular}{|c|c|c|c|c|c|}
\hline Position: x [mm] & 0 & 50 & 100 & 150 & 200 \\
\hline Time [s] & 0.181 & 0.209 & 0.222 & 0.232 & 0.250 \\
\hline
\end{tabular}

The observation of the velocity profiles in the boundary layer allows to identify the phenomena induced by the interaction with the vortex. In order to quantify these results, it is possible to determine the skin friction coefficient, which is representative of the dynamic structure of the boundary layer. It is defined as:

$$
C_{f}=\frac{\tau_{p}}{1 / 2 \rho U_{0}^{2}} \text { where the wall shear stress is given by } \tau_{p}=\mu\left(\frac{\partial u}{\partial y}\right)_{y=0}
$$

When the measurement points are not sufficiently close to the wall, it is rather difficult to precisely calculate the wall friction coefficient from the velocity profiles. Nevertheless, this method is used herein. Even if the results are not always exact, their evolution can be trusted.

Figure 6 presents the evolution of the wall friction coefficient at two different locations. It is calculated from the unsteady velocity profiles by a regression with a third order polynomial on the first six points above the plate: the shear stress is deduced from the polynomial derivative at the ordinate where it is null. The times at which the relaminarisation occurs are represented on these curves (first vertical dotted line). The second dotted line shows the time when the vortex is centred above the position of interest. 
http://dx.doi.org/10.21611/qirt.1998.034

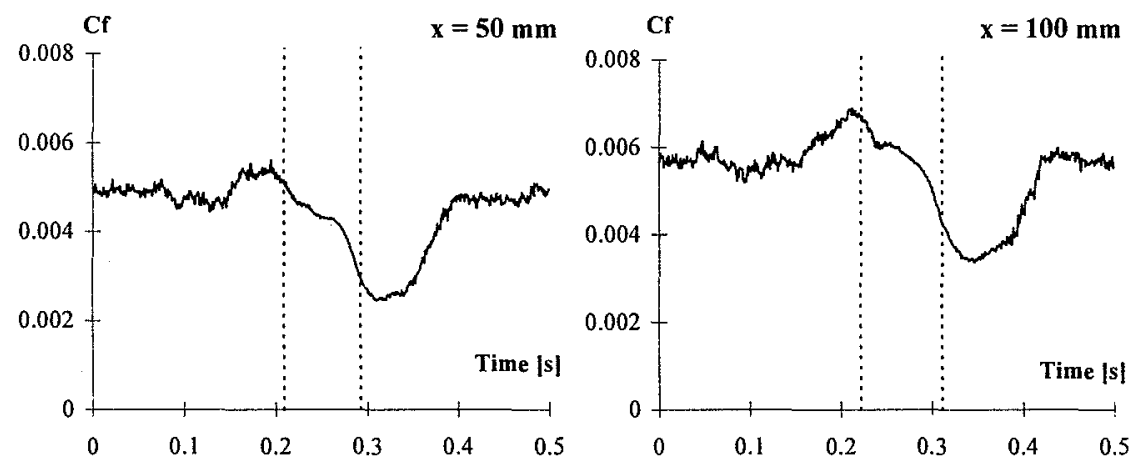

Fig. 6. Wall friction coefficient on the thermal sensor

This calculation method gives good results only when the boundary layer presents a laminar-like profile. When it is perturbed by the wake of the wing, the polynomial regression is not adapted. So, these curves are correct after the relaminarisation.

The evolution of the wall friction coefficient agrees with the preceding observations. The vortex induces a reduction of the velocities in the boundary layer, that causes the wall friction to decrease. Then the boundary layer is thickened just after the occurrence of the eddy: the wall friction presents a sharp minimum. Finally, the increase towards the initial value is accelerated by the second eddy that increases the boundary layer velocities more rapidly.

As the vortex intensity diminishes along its convection downstream, these effects are smaller. The results also confirm that, for the present configuration, no boundary layer separation occurs, the interaction parameter is rather small as compared with the values used by Doligalski and Walker [6].

\subsection{Thermal analysis}

\subsubsection{Characterisation of the mean heat transfer coefficient}

The characterisation of the convective heat transfer coefficient on the flat plate is conducted in two different places: one outside the wake of the wing and the second in its wake where the plate will be placed for the study of the interaction. The mean convective heat transfer coefficient is determined by the methodology described in paragraph 2.2.2.1. From each heat transfer map, a mean profile is calculated and represented on figure 7 where it is compared with the theoretical evolution for a laminar boundary layer given by Taine et al. [9]:

$$
N u_{x}(x)=\frac{0.324 \mathrm{Re}_{x}^{0.5} \operatorname{Pr}^{1 / 3}}{\left[1-\left(x_{0} / x\right)^{3 / / 1}\right]^{1 / 3}} \text { and } N u_{x}(x)=\frac{h_{C V}(x) x}{\lambda}
$$




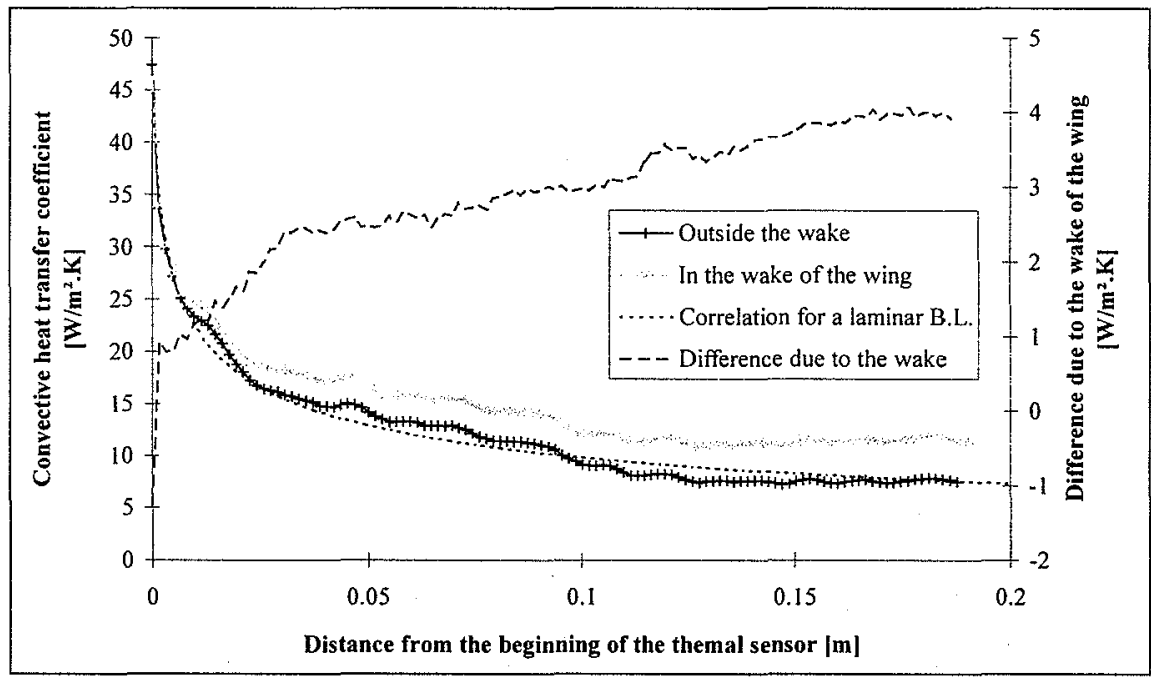

Fig. 7. Comparison of the mean heat transfer coefficient profiles with the theoretical one

When the flat plate is placed outside the wake of the wing, the experimental profile well agrees with the theoretical one. However, in the wake of the wing, the small eddies issued from its trailing edge induce an increase on the heat transfer coefficient of about $3.5 \mathrm{~W} / \mathrm{m}^{2} \cdot \mathrm{K}$.

In the present case, the radiative correction (last term in equation (1)) is not negligible:

$$
\varphi_{\text {ray }} \approx 68 W / m^{2}
$$

which represents an equivalent heat transfer coefficient:

$$
h_{\text {ray }} \approx 5.5 W / m^{2} \cdot K
$$

\subsubsection{Characterisation of the unsteady heat transfer coefficient}

The study of the heat transfer induced by the interaction between the vortex and the boundary layer is conducted by means of the procedure described in paragraph 2.2.2.2.

The infrared thermography system acquires the surface temperature along the central profile (in the flow direction) with a $500 \mathrm{~Hz}$ frequency. A synchronic average is applied over each wing motion to calculate the evolution of the temperature profile along one period with less noise (100 periods are acquired). These temperature signals are then filtered with a linear Butterworth filter (of the fourth order with a cut-off frequency of $25 \mathrm{~Hz}$ ), to reduce the high frequency non-significant noise of the thermal sensor surface temperature fluctuations. These signals are finally used as a boundary condition in the numerical simulation code that calculates the unsteady convective heat transfer coefficient between the fluid and the wall.

In figure 8 the unsteady heat transfer coefficient during one wing motion is presented. The evolution along the flow direction is the same as in the steady state case : a high heat exchange at the beginning of the heated zone $(x=0 \mathrm{~mm})$ that decreases as the thermal boundary layer thickens. In this representation, the effect of the vortex is rather small and not easily distinguished. A small diminution of the heat transfer occurs during the interaction, which decreases as the viscous dissipation reduces the vortex strength. 


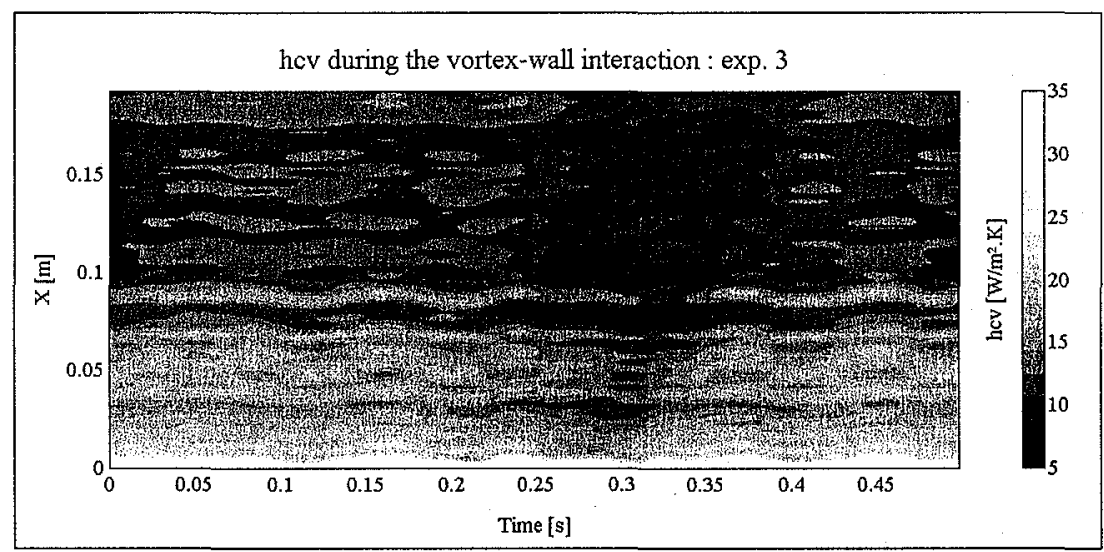

Fig. 8. Unsteady convective heat transfer coefficient: synchronic average over 100 vortex passages

In order to compare the evolution of the wall friction coefficient and the wall heat transfer coefficient, the latter is represented in figure 9 at the same abscissas as the ones of $\mathrm{Cf}$. Two experiments (2 and 3 ) with different heating conditions are shown and compared with experiment 1 that was conducted with no wing motion. The two vertical lines indicate the times corresponding to the relaminarisation and the passage of the vortex centre. The main conditions of these three experiments are listed bellow:

\begin{tabular}{|c|c|c|c|}
\cline { 2 - 4 } \multicolumn{1}{c|}{} & Experiment 1 & Experiment 2 & Experiment 3 \\
\hline Copper plate temperature & $40.0^{\circ} \mathrm{C}$ & $40.0^{\circ} \mathrm{C}$ & $60.0^{\circ} \mathrm{C}$ \\
\hline Flow temperature & $22.1^{\circ} \mathrm{C}$ & $23.9^{\circ} \mathrm{C}$ & $24.2^{\circ} \mathrm{C}$ \\
\hline
\end{tabular}

On all the curves, although the test conditions are different, experiments 2 and 3 show a similar evolution with a relatively important difference with respect with experiment 1 . Some low frequency phenomena also appear, caused by vibration of the test section. The reduction of the heat transfer occurs in two steps. The first one corresponds with the relaminarisation of the boundary layer: it is a quick variation of about $3 \mathrm{~W} / \mathrm{m}^{2} \cdot \mathrm{K}$ which is in good agreement with the difference found between the two states of the boundary layer (figure 7). Following a short plateau, just as the vortex passes above the position considered, the heat transfer reaches a minimum. This latter decrease is smaller for positions further downstream as the vortex rotation intensity diminishes. Then, the heat transfer increases again to the mean value found for the turbulent boundary layer (experiment 1 ).
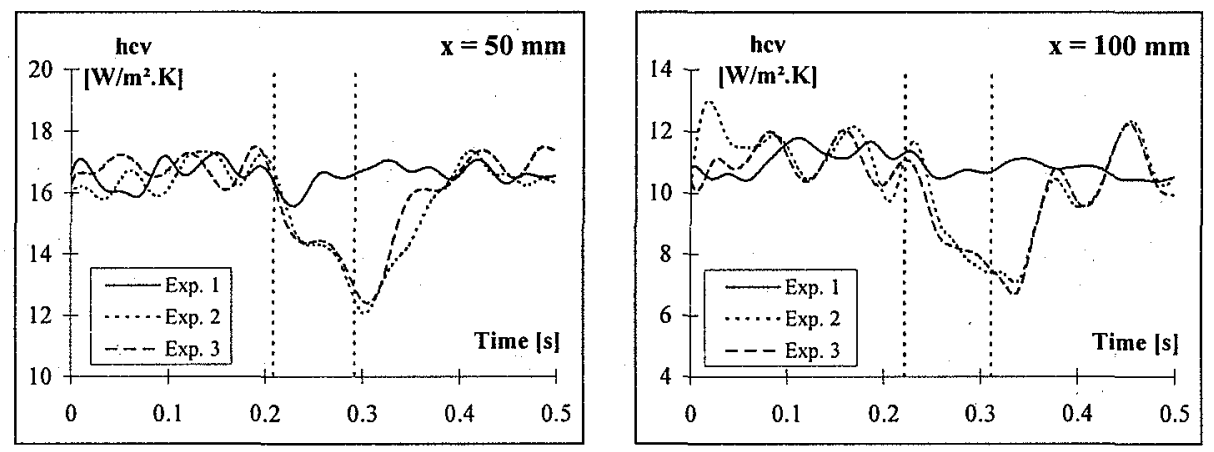

Fig. 9. Heat transfer coefficient evolution at different positions 
Even if it is rather difficult to distinguish the effects of the relaminarisation and of the vortex interaction, the results of this analysis are in good agreement with the dynamic phenomena previously described.

Two experiments ( 2 and 3 ) have been conducted for different heating conditions in order to evaluate the error on the resulting convective heat transfer coefficient. If the previous calculations are redone with an offset on the surface temperature (for example), the two calculated heat transfer coefficients corresponding to these two experiments show a very large discrepancy. The real difference between the two curves in figure 9 is smaller than $\Delta \mathrm{h}_{\mathrm{cv}}=0.5 \mathrm{~W} / \mathrm{m}^{2} \mathrm{~K}$ during the interaction period (from $\mathrm{t}=0.2 \mathrm{~s}$ to $\mathrm{t}=0.4 \mathrm{~s}$ ), so it is possible to consider that the error is less than $0.5 \mathrm{~W} / \mathrm{m}^{2} \mathrm{~K}$.

\section{Conclusion}

The processing of the velocity measurements acquired during the vortex - wall interaction has permitted to detail the instantaneous velocity fields in all the test section above the flat plate, for a good understanding of the global evolution of the vortex.

These observations have been completed by means of a second investigation of the boundary layer which shows that a relaminarisation occurs just before the vortex arrives. The interaction with the vortex is characterised by a compression of the boundary layer coupled with a strong velocity decrease near the wall, followed by a suction phenomenon inducing a thickening of the boundary layer. These effects are correlated by the evolution of the wall friction coefficient that exhibits a large diminution.

As for the heat transfer, the evolution of the thermal boundary layer is strongly coupled with the dynamic effects of the vortex. The heat transfer coefficient shows a sharp decrease because of the thickening of the boundary layer and the lower velocities of the flow near the wall.

\section{REFERENCES}

[1] BOOTH (E. R.), YU (J. C.) - Two dimensional blade - vortex interaction. Flow visualisation investigation, AlAA paper $n^{\circ} 84-2307,1984$.

[2] WALKER (J. M.), HELIN (H. E.), STRICKLAND (J. H.) - An experimental investigation of an airfoil undergoing large amplitude motions, AIAA Journal Vol. 23, nº, 1985.

[3] FRANCIS (M. S.), KEESEE (J. E.) - Airfoil dynamic stall performance with large amplitude motions, AIAA Journal Vol. 23, n¹1, 1985.

[4] PEUBE (J.), FERRET (B.) - Réalisation et caractérisation d'un tourbillon reproductible, destiné à étudier des phénomènes instationnaires dans un cadre bidimensionnel, La Recherche Aérospatiale, $n^{\circ} 4,23-36,1991$.

[5] REULET (P.), DUMOULIN (J.), MILLAN (P.) - Identification du coefficient de transfert de chaleur pariétal instationnaire, Congrès SFT, Toulouse, 1997.

[6] DOLIGALSKI (T. L.), WALKER (J. D. A.) - The boundary layer induced by a convected two-dimensional vortex, J. Fluid Mech. vol. 139, pp 1-28, 1984.

[7] ESCRIVA (X.) - Ecoulement instationnaire bidimensionnel incompressible dans un canal équipé de perturbateurs périodiques, DEA report, Université Paul Sabatier Toulouse, 1995.

[8] REULET (P.), ESCRIVA (X.), MARCHAND (M.), GIOVANNINI (A.), MILLAN (P.) - Etude locale instationnaire des coefficients d'échanges pariétaux pour des interactions tourbillon - paroi, Congrès SFT, Toulouse, 1997.

[9] TAINE (J.), PETIT (J. P.) - Transferts thermiques, mécaniques des fluides anisotropes. Dunod Université, 1989. 